\title{
Peristaltic transport of a power-law fluid in a porous tube
}

\author{
A. RAMACHANDRA RAO * and MANORANJAN MISHRA \\ Department of Mathematics, \\ Indian Institute of Science, Bangalore-560012, INDIA. \\ Email: ramchand@math.iisc.ernet.in, mishra@math.iisc.ernet.in
}

\begin{abstract}
Peristaltic transport of a power-law fluid in an axisymmetric porous tube is studied under long wavelength and low Reynolds number assumptions. The slip boundary conditions given by Beavers-Joseph and Saffman type are considered in obtaining solutions for the flow and resulting pumping characteristics are compared. Trapping and reflux phenomena are discussed for various parameters of interest governing the flow like $D a$ Darcy number, $\alpha$ Beavers-Joseph constant and $n$ the fluid behavior index. The novel feature arising in pumping due to a straight section dominated (SSD) wave form other than sinusoidal wave is discussed. The time mean flow becomes negative in free pumping for a shear thickening fluid or shear thinning fluid for an expansion or contraction SSD wave respectively. The pressure rise increases for the increasing of $D a$ against which the peristalsis acts as a pump and decreases for an increase in $\alpha$. Peristalsis works as a pump against a greater pressure rise for a shear thickening fluid and the opposite happens for a shear thinning fluid, compared with Newtonian fluid. The trapped bolus volume for sinusoidal wave is observed to decrease as the fluid behavior index decreases from shear thickening to shear thinning fluid whereas it increases for increasing Darcy number. The rheological property of the fluid, wave shape and porous nature of the wall play an important role in peristaltic transport and may be useful in understanding transport of chyme in small intestines.
\end{abstract}

Keywords: Peristaltic pumping, power-law fluid, porous medium, trapping, reflux.

\section{Introduction}

Peristalsis is an important mechanism for mixing and transporting fluids, which is generated by a progressive wave of contraction or expansion moving on the wall of the tube. The mechanism is found in the gastrointestinal, urinary, reproductive tracts and many other glandular ducts in a living body. Considerable analysis of this mechanism has been carried out, primarily for a Newtonian fluid with a periodic train of sinusoidal peristaltic waves. The inertia free peristaltic flow with long wavelength analysis was given by Shapiro et al. [1]. The early developments on mathematical modeling and experimental fluid mechanics of peristaltic flow was given in a comprehensive review by Jaffrin and Shapiro [2].

\footnotetext{
${ }^{*}$ Corresponding author: E-mail: ramchand@math.iisc.ernet.in (A. R. Rao), Fax: +91-80-3600146.
} 
The main features of the peristaltic pumping, such as trapping and reflux phenomena, have been studied extensively for Newtonian fluids. However, the rheological properties of the fluids can affect these characteristics significantly. Further, many of the physiological fluids are known to be non-Newtonian. Peristaltic transport of blood in small vessels was investigated using the viscoelastic, power-law, Casson, micropolar fluid models by $[3,4,5,6]$ respectively. Peristaltic flow of a second-order fluid in a planar channel and in an axisymmetric tube is studied by Siddiqui et al. $[7,8]$ under long-wavelength assumption. The power-law model was used to study the fluid transport in the male reproductive tract by [9], small intestine and oesophagus by $[10,11]$. Peristaltic pumping of two-layered power-law fluids was investigated by $[12,13,14]$. However, the stress-strain relationship taken in $[5,9]$ for the peristaltic transport of power-law fluid (Ostwald-de Waele type) is valid only when the rate of shear is negative and if it is positive a real solution does not exist as the fluid behavior index $n$ takes any positive real number. The important characteristics of peristalsis such as trapping and reflux phenomena are not studied in most of the above literature.

The gastrointestinal tract is surrounded by a number of heavily innervated smooth muscle layers, contraction of these muscle layers can mix the contents of the tract and move food in a controlled manner in an appropriate direction, [15]. Epithelial cells, beneath these layers are responsible for the absorption of nutrients and water from the intestine. These layers consists of many folds and there are pores through the tight junctions of them. Motivated by these facts, peristaltic motion of a power-law fluid is investigated in an axisymmetric tube with porous material at the boundary. A model coated with a porous layer at the wall for a Newtonian fluid was studied by Reese and Rath [16] in order to model the transport of synovial fluid in a knee joint. The boundary conditions to be satisfied at the interface between a porous medium and fluid layer are the matching of velocity and stresses. Beavers and Joseph [17] were the first to investigate the fluid flow at the interface between a porous medium and fluid layer in an experimental study and proposed a slip boundary conditions at the interface. A theoretical justification, using a statistical approach, of the boundary conditions of Beavers-Joseph was given by Saffman [18] and proposed an improved boundary condition.

In this paper the peristaltic transport of a power-law fluid (Ostwald-de Waele type)[19] which accommodates the study of both shear thinning and shear thickening fluids in an axisymmetric porous-tube is investigated under long-wavelength and inertia free approximations. This nonNewtonian fluid model is one of the simplest and most widely used. Food stuffs like banana puree, tomato paste and apple sauce are observed to fit this power-law model (Cheremisinoff[20], Usha and Ramachandra Rao [12]). They have presented the corresponding fluid consistency and behavior index parameters at different temperatures. As the wall of the tube is made up of porous material, slip boundary conditions of both Beavers-Joseph and Saffman type are considered to obtain the solutions. It is assumed that the Darcy's law holds inside the porous medium which is saturated with a fluid of power-law type. The effects of straight section dominated(SSD) wave forms on peristaltic pumping are discussed. A negative mean flow is achieved under free pumping for a shear thickening fluid with a SSD expansion wave and the same is observed for a SSD contraction wave in the case of a shear thinning fluid. These results are similar to those of Provost and Schwarz [21], who observed that whenever the fluid deviated from Newtonian 
behavior there exists a nontrivial peristaltic wave form for which the time averaged flux is zero or negative in the absence of a mean pressure gradient. Peristaltic pumping, trapping and reflux phenomena are discussed for various parameters of interest governing the flow.

\section{Mathematical formulation}

Consider the peristaltic transport of an incompressible power-law fluid in an axisymmetric tube, shown in Fig. 1. A porous material saturated with a power-law fluid with a consistency different from that of the inside power-law fluid but having the same fluid behavior index $n$ forms the tube boundary. The mean radius of the tube is $a$. The wall of the tube is flexible, which is an interface of the fluid and porous medium. The wall is subjected to a periodic peristaltic wave movement with wave speed $c$, wavelength $\lambda$ and amplitude $b$. The instantaneous radius at any axial station $Z$ is given by $R=H(Z-c t)$, where $t$ is time. The flow becomes steady in a wave frame $(r, z)$ moving away with speed $c$ in the direction of the wave from a fixed frame $(R, Z)$ given by

$$
z=Z-c t, r=R, u=U-c, v=V, p(x)=P(X, t),
$$

under the assumptions that the pressure difference across a wavelength is constant and the length of the tube is an integral multiple of the wavelength. Here $(u, v)$ and $(U, V)$ are the velocity components in axial and radial directions, $p$ and $P$ are the pressures in wave and fixed frame of references respectively.

We choose the Ostwald-de Waele power-law model which is characterized by the constitutive equation [19]

$$
\tau=-m_{1}\left\{\left|\sqrt{\frac{1}{2}(\Delta: \Delta)}\right|^{n-1}\right\} \Delta .
$$

Here $\tau$ is the stress tensor, $\Delta$ is the symmetric rate of deformation tensor,

$$
\frac{1}{2}(\Delta: \Delta)=2\left\{\left(\frac{\partial v}{\partial r}\right)^{2}+\left(\frac{v}{r}\right)^{2}+\left(\frac{\partial u}{\partial z}\right)^{2}\right\}+\left(\frac{\partial u}{\partial r}+\frac{\partial v}{\partial z}\right)^{2},
$$

$m_{1}$ and $n$ are respectively the consistency and fluid behavior index parameters. A shear thinning or pseudo plastic fluid is characterized by the fluid behavior index $n<1$, and $n>1$ corresponds to a shear thickening or dilatant fluid. For $n=1$, the Eq. (2.2) reduces to the usual Newtonian model.

The equations of motion of a power-law fluid in an axisymmetric tube are given by

$$
\left.\begin{array}{l}
\rho\left(u \frac{\partial u}{\partial z}+v \frac{\partial u}{\partial r}\right)=-\frac{\partial p}{\partial z}-\left(\frac{1}{r} \frac{\partial\left(r \tau_{r z}\right)}{\partial r}+\frac{\partial \tau_{z z}}{\partial z}\right), \\
\rho\left(u \frac{\partial v}{\partial z}+v \frac{\partial v}{\partial r}\right)=-\frac{\partial p}{\partial r}-\left(\frac{1}{r} \frac{\partial\left(r \tau_{r r}\right)}{\partial r}+\frac{\partial \tau_{r z}}{\partial z}\right) .
\end{array}\right\}
$$

Introducing the non-dimensional quantities

$$
z^{\prime}=\frac{z}{\lambda}, r^{\prime}=\frac{r}{a}, t^{\prime}=\frac{c t}{\lambda}, p^{\prime}=\frac{a^{n+1} p}{m_{1} \lambda c^{n}}, u^{\prime}=\frac{u}{c}, v^{\prime}=\frac{v}{c \delta}, \delta=\frac{a}{\lambda}, h=\frac{H}{a}, \phi=\frac{b}{a}, m=\frac{m_{2}}{m_{1}},
$$


where $m_{2}$ is the consistency parameter of the fluid in the porous medium, and the components of stress in Eq. (2.3)(dropping the primes), we get

$$
\left.\begin{array}{rl}
\operatorname{Re} \delta\left(u \frac{\partial u}{\partial z}+v \frac{\partial u}{\partial r}\right) & =-\frac{\partial p}{\partial z}+\frac{1}{r} \frac{\partial}{\partial r}\left\{\Phi\left(r \frac{\partial u}{\partial r}+\delta^{2} 4 \frac{\partial v}{\partial z}\right)\right\}+2 \delta^{2} \frac{\partial}{\partial z}\left(\Phi \frac{\partial u}{\partial z}\right), \\
\operatorname{Re} \delta^{3}\left(u \frac{\partial v}{\partial z}+v \frac{\partial v}{\partial r}\right) & =-\frac{\partial p}{\partial r}+\delta^{2} \frac{1}{r} \frac{\partial}{\partial r}\left(\Phi r \frac{\partial v}{\partial r}\right)+\delta^{2} \frac{\partial}{\partial z}\left\{\Phi\left(\frac{\partial u}{\partial r}+\delta^{2} \frac{\partial v}{\partial z}\right)\right\} .
\end{array}\right\}
$$

where $\Phi=\left|\sqrt{2 \delta^{2}\left\{\left(\frac{\partial v}{\partial r}\right)^{2}+\left(\frac{v}{r}\right)^{2}+\left(\frac{\partial u}{\partial z}\right)^{2}\right\}+\left(\frac{\partial u}{\partial r}+\delta^{2} \frac{\partial v}{\partial z}\right)^{2}}\right|^{n-1}$ and $R e=\rho a^{n} / m_{1} c^{n-2}$, Reynolds number for power-law fluid [22]. Under the assumptions of long wavelength, $\delta<<1$ and low Reynolds number $R e \rightarrow 0$ (negligible inertia), the governing Eq. (2.4) become

$$
\left.\begin{array}{l}
\frac{\partial p}{\partial z}=\frac{1}{r} \frac{\partial}{\partial r}\left(r \frac{\partial u}{\partial r}\left|\frac{\partial u}{\partial r}\right|^{n-1}\right), \\
\frac{\partial p}{\partial r}=0 .
\end{array}\right\}
$$

The flow in the porous medium is governed by the Darcy's law and following Shenoy [23], the non-dimensional Darcy's law of power-law fluid is given by

$$
\left|u_{\text {por }}\right|^{n-1} u_{\text {por }}=-\frac{D a}{m} \frac{\partial p}{\partial z}
$$

where $u_{p o r}$ is the velocity in the porous medium, $D a=\frac{k}{a^{n+1}}$, Darcy number, $k$ is a modified permeability and its dimension is length to the power $n+1$ due to the power-law behavior of the fluid.

\subsection{Boundary conditions}

The boundary is an interface which separates the power-law fluid and the porous medium. Here, we present solutions for two types of slip boundary conditions and try to compare the effects of these conditions on the flow characteristics.

Beavers-Joseph boundary conditions for a power-law fluid are given by (in a waveframe of reference):

$$
\left.\begin{array}{rlrl}
u & =u_{h}-1, & & \text { at } r=h(z), \\
\frac{\partial u}{\partial r} & =\frac{\alpha}{D a^{\frac{1}{n+1}}}\left(u_{h}-u_{p o r}\right), & & \text { at } r=h(z), \\
\frac{\partial u}{\partial r} & =0, & & \text { at } r=0,
\end{array}\right\}
$$

where $u_{h}$ is the slip velocity at the boundary $r=h(z), \alpha$ is dimensionless Beavers-Joseph constant which depends on the nature of the porous medium but not on the fluid viscosity.

The boundary conditions corresponding to Saffman are given by:

$$
\left.\begin{array}{rlrl}
u & =\frac{D a^{\frac{1}{n+1}}}{\alpha} \frac{\partial u}{\partial r}-1, & & \text { at } r=h(z), \\
\frac{\partial u}{\partial r} & =0, & & \text { at } r=0 .
\end{array}\right\}
$$

The third condition of (2.7) and second condition of (2.8) imply that the velocity is maximum on the axis of the tube. 


\section{Solutions}

\subsection{Beavers-Joseph model}

From Eq. (2.6), the velocity of porous medium is $u_{p o r}=-\left(\frac{D a}{m}\right)^{1 / n} \frac{\partial p}{\partial z}\left|\frac{\partial p}{\partial z}\right|^{\frac{1}{n}-1}$. Integrating the Eqs. (2.5) using boundary conditions (2.7), we get

$$
u=\frac{\partial p}{\partial z}\left|\frac{\partial p}{\partial z}\right|^{\frac{1}{n}-1}\left\{\left(\frac{1}{2}\right)^{\frac{1}{n}} \frac{n}{n+1}\left(r^{\frac{1}{n}+1}-h^{\frac{1}{n}+1}\right)\right\}+u_{h}-1
$$

where

$$
u_{h}=\frac{\partial p}{\partial z}\left|\frac{\partial p}{\partial z}\right|^{\frac{1}{n}-1}\left\{\frac{D a^{\frac{1}{n+1}}}{\alpha}\left(\frac{h}{2}\right)^{\frac{1}{n}}-\left(\frac{D a}{m}\right)^{\frac{1}{n}}\right\} .
$$

The solution in terms of stream function $\left(u=\frac{1}{r} \frac{\partial \psi}{\partial r}, v=-\frac{1}{r} \frac{\partial \psi}{\partial z}\right)$ is obtained from Eq. (3.1) by using the condition $\psi=0$ at $r=0$, as

$$
\begin{array}{r}
\psi=\frac{r^{2}}{2}\left[-1+\frac{\partial p}{\partial z}\left|\frac{\partial p}{\partial z}\right|^{\frac{1}{n}-1}\left\{\left(\frac{1}{2}\right)^{\frac{1}{n}}\right.\right. \\
\frac{n}{n+1}\left(\frac{2 n}{1+3 n} r^{\frac{1}{n}+1}-h^{\frac{1}{n}+1}\right) \\
\left.\left.+\frac{D a^{\frac{1}{n+1}}}{\alpha}\left(\frac{h}{2}\right)^{\frac{1}{n}}-\left(\frac{D a}{m}\right)^{\frac{1}{n}}\right\}\right]
\end{array}
$$

The non-dimensional flow rate $q$ across any cross section of tube is independent of $z$ under lubrication approach and is given by

$$
\begin{aligned}
q & =2 \int_{0}^{h} r u d r \\
& =h^{2}\left[-1+\frac{\partial p}{\partial z}\left|\frac{\partial p}{\partial z}\right|^{\frac{1}{n}-1}\left\{\frac{D a^{\frac{1}{n+1}}}{\alpha}\left(\frac{h}{2}\right)^{\frac{1}{n}}-\left(\frac{D a}{m}\right)^{\frac{1}{n}}-\left(\frac{1}{2}\right)^{\frac{1}{n}} \frac{n}{1+3 n} h^{\frac{1}{n}+1}\right\}\right] .
\end{aligned}
$$

Inverting the absolute value appropriately Eq.(3.4) is rewritten as

$$
\begin{aligned}
\frac{\partial p}{\partial z}= & \left(\frac{1+3 n}{n}\right)^{n} \frac{2}{h^{2 n}} \\
& \left(\frac{q+h^{2}}{\frac{1+3 n}{n}\left(\frac{D a^{\frac{1}{n+1}}}{\alpha} h^{\frac{1}{n}}-\left(\frac{2 D a}{m}\right)^{\frac{1}{n}}\right)-h^{\frac{n+1}{n}}}\right)\left|\frac{q+h^{2}}{\frac{1+3 n}{n}\left(\frac{D a^{\frac{1}{n+1}}}{\alpha} h^{\frac{1}{n}}-\left(\frac{2 D a}{m}\right)^{\frac{1}{n}}\right)-h^{\frac{n+1}{n}}}\right|^{n-1}
\end{aligned}
$$

\subsection{Saffman model}

Integrating the Eq. (2.5) using Saffman boundary conditions (2.8), we get

$$
u=-1+\left(\frac{1}{2}\right)^{\frac{1}{n}} \frac{\partial p}{\partial z}\left|\frac{\partial p}{\partial z}\right|^{\frac{1}{n}-1}\left\{\frac{n}{n+1}\left(r^{\frac{1}{n}+1}-h^{\frac{1}{n}+1}\right)+\frac{D a^{\frac{1}{n+1}}}{\alpha} h^{\frac{1}{n}}\right\},
$$


and the solution in terms of the stream function is

$$
\psi=\frac{r^{2}}{2}\left[-1+\left(\frac{1}{2}\right)^{\frac{1}{n}} \frac{\partial p}{\partial z}\left|\frac{\partial p}{\partial z}\right|^{\frac{1}{n}-1}\left\{\frac{n^{2}}{(n+1)(1+3 n)}\left(2 r^{\frac{1}{n}+1}-\frac{1+3 n}{n} h^{\frac{1}{n}+1}\right)+\frac{D a^{\frac{1}{n+1}}}{\alpha} h^{\frac{1}{n}}\right\}\right] .
$$

The corresponding flow rate $q$ is given by

$$
q=h^{2}\left[-1+\left(\frac{h}{2}\right)^{\frac{1}{n}} \frac{\partial p}{\partial z}\left|\frac{\partial p}{\partial z}\right|^{\frac{1}{n}-1}\left\{\frac{D a^{\frac{1}{n+1}}}{\alpha}-\frac{n}{1+3 n} h\right\}\right],
$$

and this implies

$$
\frac{\partial p}{\partial z}=\frac{2}{h^{1+2 n}}\left(\frac{q+h^{2}}{\frac{D a^{\frac{1}{n+1}}}{\alpha}-\frac{n}{1+3 n} h}\right)\left|\frac{q+h^{2}}{\frac{D a^{\frac{1}{n+1}}}{\alpha}-\frac{n}{1+3 n} h}\right|^{n-1} .
$$

In the limit $D a \rightarrow 0$, the solutions given in Eqs. (3.1-3.9) reduce to the solutions with no-slip boundary condition obtained by Usha and Ramachandra Rao [12] with parameters $k_{1}=k_{2}=k$ and $m=1$ for a single power-law fluid. Further, when the fluid behavior index $n=1$ and $D a \rightarrow 0$, our results reduce to the corresponding results for a viscous fluid given by Shapiro et al. [1].

Srivastava and Srivastava $[9,10]$ have used the constitutive equation for Ostwald-de Waele power-law fluid model without any absolute value sign which may not be correct as the rate of deformation tensor takes both positive and negative values. Their relation is valid only when $\partial u / \partial r$ is positive and when it is negative no real solution exists. Radhakrishnamacharya [4] has considered a proper constitutive equation for Ostwald-de Waele power-law fluid model to start with, but the absolute sign was missed while considering the various orders equations leading to incorrect solutions.

The dimensionless time averaged flux $\bar{Q}$ in the fixed frame is obtained as

$$
\bar{Q}=\frac{2}{T} \int_{0}^{T} \int_{0}^{H} R U d R d t=2 \int_{0}^{1} \int_{0}^{h} r(u+1) d r d z=q+\int_{0}^{1} h^{2} d z .
$$

\section{Discussion of Results}

\subsection{Pumping characteristics}

We study the pumping characteristics of a power-law fluid in a tube with consistency parameter $\mathrm{m}=1$, by choosing three types of non-dimensional wave forms, namely sinusoidal, expansion (+sign) and contraction (-sign) waves, given by

$$
\left.\begin{array}{rlrl}
h(x) & =1+\phi \sin (2 \pi x) & & 0 \leq x \leq 1, \\
h(x) & =1 \pm \phi \sin \left(\pi x / \lambda_{c}\right) & & 0 \leq x \leq \lambda_{c} \\
& =1 & & \lambda_{c} \leq x \leq 1 .
\end{array}\right\}
$$

The wave of expansion or contraction given in Eq. (4.2) is confined to a portion of length $\lambda_{c}$ and remains same over the rest of the wavelength giving straight section. The integral in Eq. (3.10), 
depends on the wave form one chooses. It takes a value $1+\frac{\phi^{2}}{2}$ and $1+\frac{\phi^{2} \lambda_{c}}{2} \pm \frac{4 \phi \lambda_{c}}{\pi}$ for sinusoidal, expansion (+sign) and contraction (-sign) wave forms respectively. Substituting $q$ from Eq. (3.10) in Beavers-Joseph solution Eq. (3.5) or Saffman solution Eq. (3.9) and integrating $\frac{\partial p}{\partial z}$ with respect to $z$ over one wavelength yields a relation between $\bar{Q}$ and $\Delta p$ as

$$
\Delta p=p_{1}-p_{0}=\int_{0}^{1} \frac{\partial p}{\partial z} d z=f(\bar{Q}, D a, \alpha, n, \phi) .
$$

The variation of $\bar{Q}$ with $\Delta p$ is given by evaluating the integral (4.3) for both Beavers-Joseph or Saffman models, using the numerical quadrature formulae of the Matlab software package.

In Fig. 2 the variation of $\bar{Q}$ with $\Delta p$ is shown for different $D a$ and $\alpha$ by fixing the other parameters $n=1$ and $\phi=0.6$, for a sinusoidal wave form given in Eq. (4.1). It is observed from Fig 2(i) that increasing $D a$ from 0(no slip) to a small positive number (slip flow), gives a better pumping performance $(\Delta p>0$, in pumping region) as well as in free pumping $(\Delta p=0)$. The dashed lines in the figure correspond to Saffman model and they coincide with the BeaversJoseph model for small $D a\left(=10^{-5}, 10^{-4}\right)$ where as for large $D a\left(=10^{-3}\right)$ pumping is more for Saffman model. The effect of the parameter $\alpha$ on pumping characteristics is plotted in Fig. 2(ii) for $\phi=0.6, D a=10^{-4}, n=1$. It is observed that, increasing $\alpha$ decreases the pumping as well as free pumping and there is no difference between the Beavers-Joseph and Saffman models on pumping as the pumping curves coincide with each other.

The effects of power-law behavior index $\mathrm{n}$ on pumping with slip and no-slip boundary conditions are depicted for the sinusoidal wave shape (Eq. (4.1)) in Fig. 3. The pressure rise required to produce zero time-averaged flux is denoted by $\Delta p_{0}$ and when $\Delta p>\Delta p_{0}, \bar{Q}$ is negative. The variation of $\Delta p_{0}$ with $n$ for $\phi=0.6, \alpha=0.5$ is plotted in Fig. 3(i) for different values of $D a$. It is observed that $\Delta p_{0}$ increases with an increase of fluid behavior index $n$, indicating that the pumping increases as the fluid changes from shear thinning $(n<1)$ to Newtonian $(n=1)$ and to shear thickening $(n>1)$. Further, as $D a$ increases $\Delta p_{0}$ also increases only for large value of $n$, which means that the pumping is better for shear thickening fluid and the effects of slip are not significant for small values of $n<1$ for a shear thinning fluid. For $D a \geq 10^{-4}$, the pumping characteristics are the same for Beavers-Joseph and Saffman models for a shear thinning fluid but little differences are seen at larger values of $n$ for shear thickening fluid. The variation of $\Delta p_{0}$ with $n$ for $\phi=0.6$ and $D a=10^{-4}$ for different $\alpha$ is shown in Fig. 3(ii). Increasing values of $\alpha$ one observes significant decrease in pumping for large values of $n$, similar to the effects of $D a$ as seen from Fig. 3(i). Saffman model gives a better pumping performance than Beavers-Joseph model for a shear thickening fluid compared to shear thinning fluid and Newtonian fluid. Thus, we conclude that the effect of slip condition is more prominent in the shear thickening fluid than in shear thinning fluid or Newtonian fluid.

In order to observe the influence of the rheological properties of the fluid for different wave shapes, we have plotted in Fig. 4 the pumping characteristics for a SSD expansion or contraction waveforms given in Eq. (4.2). Figure 4(i) shows the variation of $\bar{Q}$ with $\Delta p$ for a contraction wave for no-slip boundary conditions $D a=0$, and $\phi=0.4, \lambda_{c}=0.2$. An interesting observation is that for a specific parameter of shear thinning fluid $(n<0.4)$, free pumping results in negative mean flow. The pressure rise required to produce zero mean flow, $\Delta p_{0}$, becomes negative where 
as $\Delta p_{0}$ is always positive for any non-Newtonian fluid with sinusoidal waveform. Figure 4(ii) depicts the effects of expansion wave shape on pumping characteristics of power-law fluids with $D a=0, \phi=0.4$, and $\lambda_{c}=0.2$. It is seen that for some shear thickening fluid with $n \geq 3$, the free pumping results in negative mean flow and $\Delta p_{0}$ becomes negative. Provost and Schwarz [21], Usha and Rao [12] have also observed similar things for power-law fluid models.

The effects of slip flow with different $\alpha$ of Beavers-Joseph model for fixed $D a=10^{-5}, \phi=$ $0.3, \lambda_{c}=0.2, n=2$ for an expansion wave is plotted in Fig. 4(iii) and it is observed that a negative free pumping flux or negative $\Delta p_{0}$ is possible only for large values of $\alpha$. Whereas, for a contraction wave $\Delta p_{0}$ never becomes negative for any value of $\alpha$. To understand the effect of SSD on pumping, we have plotted the variation of $\Delta p_{0}$ with $\lambda_{c}$ for a contraction wave (Fig. 5(i)) and an expansion wave (Fig. 5(ii)) for different values of $n$ with $D a=10^{-5}, \alpha=0.5$ and $\phi=0.4$. As expected when $\lambda_{c}$ increases the straight section domination reduces. From the figures we observe that $\Delta p_{0}$ is negative for small values of $\lambda_{c}$, when $n<1$ for a contraction wave and when $n>1$ for an expansion wave. Further, for an expansion wave form pumping decreases with an increasing $n$ for small $\lambda_{c}$ where as when the straight section domination reduces (i.e. when $\lambda_{c}$ is large) the pumping increases with an increasing $n$ as seen from Fig. 5(ii).

\subsection{Trapping}

The formation of an internally circulating bolus of fluid by closed streamlines is called trapping, and this trapped bolus is pushed ahead along with the peristaltic wave. A criterion for central streamline trapping is the existence of stagnation points where both the velocity components $u$ and $v$ vanish in the wave frame and they are located at the intersections of the curve $\psi=0$ and the centerline. From the Eqs. (3.1) and (3.4), the stagnation points for Beaver-Joseph model are given by

$$
q-\frac{2 n h^{\frac{1}{n}+3}}{(3 n+1)\left\{\frac{n+1}{n}\left(\frac{D a^{\frac{1}{n+1}} h^{\frac{1}{n}}}{\alpha}-\left(\frac{2 D a}{m}\right)^{\frac{1}{n}}\right)-h^{\frac{1}{n}+1}\right\}}=0 .
$$

The stagnation points are real if the roots $h$ of Eq. (4.4) lie in the interval $1-\phi \leq h \leq 1+\phi$. The value of $q$ corresponding to the limiting cases $h=1+\phi$ and $1-\phi$ are the lower and upper trapping limits on the flux respectively. The corresponding trapping limits for the time averaged flux can be obtained for a general wave shape using the Eq. (3.10). The center line trapping limits $\left(\bar{Q}_{l}<\bar{Q}<\bar{Q}_{m}\right)$ for a sinusoidal wave form (4.1) are given by

$$
\left.\begin{array}{rl}
\bar{Q}_{l} & =1+\frac{\phi^{2}}{2}+\frac{2 n(1+\phi)^{\frac{1}{n}+3}}{(3 n+1)\left\{\frac{n+1}{n}\left(\frac{D a^{\frac{1}{n+1}}(1+\phi)^{\frac{1}{n}}}{\alpha}-\left(\frac{2 D a}{m}\right)^{\frac{1}{n}}\right)-(1+\phi)^{\frac{1}{n}+1}\right\}}, \\
\bar{Q}_{m} & =1+\frac{\phi^{2}}{2}+\frac{2 n(1-\phi)^{\frac{1}{n}+3}}{(3 n+1)\left\{\frac{n+1}{n}\left(\frac{D a^{\frac{1}{n+1}}(1-\phi)^{\frac{1}{n}}}{\alpha}-\left(\frac{2 D a}{m}\right)^{\frac{1}{n}}\right)-(1-\phi)^{\frac{1}{n}+1}\right\}} .
\end{array}\right\}
$$


Similarly the trapping limits corresponding to Saffman model are obtained as

$$
\left.\begin{array}{rl}
\bar{Q}_{l} & =1+\frac{\phi^{2}}{2}+\frac{2 n(1+\phi)^{3}}{(3 n+1)\left(\frac{n+1}{n} \frac{D a}{n+1}-1-\phi\right)}, \\
\bar{Q}_{m} & \left.=1+\frac{\phi^{2}}{2}+\frac{2 n(1-\phi)^{3}}{(3 n+1)\left(\frac{n+1}{n} \frac{D a}{n+1}\right.}-1+\phi\right)
\end{array}\right\}
$$

These limits reduce to those given by Shapiro et al. [1] for a Newtonian fluid, in the limit $D a \rightarrow 0$ and $n=1$.

In what follows we discuss the streamline patterns for Beavers-Joseph model for a sinusoidal wave form. The streamline patterns in the waveframe for $\bar{Q}=0.1$, with $\alpha=0.5, \phi=0.4$, and $n=1$ for different values of $D a$ are shown in Fig. 6. The trapped bolus doesn't exist for the flux $\bar{Q}$ chosen in Fig. 6(i) for $D a=0$ (no-slip condition), but the trapping exists when $D a \neq 0$ and the bolus volume increases with an increase in $D a$ i.e. the pore size increases, which is seen in Figs. 6(ii) and 6(iii). The streamlines with $\bar{Q}=0.1, \phi=0.4, D a=10^{-4}$ and $n=1$ for different $\alpha$ are plotted in Fig. 7. It is observed that the size of the trapped bolus decreases with increasing $\alpha$. The effect of power-law fluid behavior index $n$ on streamline patterns is depicted in Fig. 8, for $\bar{Q}=0.3, \phi=0.4, D a=10^{-4}$, and $\alpha=0.5$. The bolus size decreases as the fluid behavior index $n$ decreases and disappears for $n=0.3$, as seen in Fig. 8(iv). In all the above discussions the flux $\bar{Q}$ is chosen in pumping range and we observe that the increase in pumping corresponds to an increase in the trapped bolus size. The streamlines in copumping range fluxes produce similar results as in [24], where the trapped bolus shifts towards the boundary and they are not shown here.

\subsection{Reflux}

The presence of some fluid particles whose mean motion over one cycle is against the net pumping direction is known as reflux. This phenomenon is of physiological importance as it implies a possible backward motion of bacteria against the direction in which physiological fluids are pumped. Shapiro et al. [1] have emphasized that the reflux phenomenon should be studied in the fixed frame of reference using the Lagrangian method. But in wave frame the streamlines and particle trajectories coincide. We may therefore employ the value of stream functions in the waveframe as a means of identifying material particles. Following [1], $Q_{\psi}$ defined as dimensionless volume flow rate in the fixed frame between the axis of the tube and the wave frame streamline $\psi$. This is an indicator of the material particles in fixed frame, and is given by

$$
Q_{\psi}=2 \int_{0}^{R(\psi, Z, t)} R U(R, Z, t) d R .
$$

By using the transformation between the two frames of reference given in Eq. (2.1), we get $Q_{\psi}=2 \psi+r^{2}(\psi, z, t)$. Averaging it over one period of the wave, we get

$$
\bar{Q}_{\psi}=2 \psi+\int_{0}^{1} r^{2}(\psi, z) d z
$$


Now, we define $Q_{\psi}^{*}=\bar{Q}_{\psi} / \bar{Q}_{w}, \psi^{*}=\psi / \psi_{w}$, where $\bar{Q}_{w}$ and $\psi_{w}$ are the values of $\bar{Q}_{\psi}$ and $\psi$ at the wall $(r=h), \bar{Q}_{w}=\bar{Q} / 2$, and $\psi_{w}=q / 2$. If $Q_{\psi}^{*}$ increases with an increase in $\psi^{*}$ then the motion of the particles is always in the pumping direction, and if $Q_{\psi}^{*}$ decreases with increasing $\psi^{*}$ then reflux appears in that region. It is shown in [1] for a single fluid that a reflux layer exists near the wall whenever $Q_{\psi}^{*}$ increases to a value greater than unity and decreases to unity at the wall.

Here, we discuss the reflux phenomena for Beavers-Joseph model with a sinusoidal peristaltic wave. The Eq. (3.3) is solved numerically for $r$ as a function of $z$, for a given value of constant $\psi$ and the integral in (4.8) is evaluated by using a numerical quadrature formulae of Matlab package. The effects of porous wall and the fluid behavior index on reflux phenomena are plotted in Fig. 9. Figure 9(i) shows the variation of $Q_{\psi}^{*}$ as a function of $\psi^{*}$ for different values of $D a$ fixing $\bar{Q}=0.915, \alpha=0.5, \phi=0.6$ and $n=1$. The variation of $Q_{\psi}^{*}$ for $D a=0$ is a strictly increasing function of $\psi^{*}$ and attains unity only at the wall, which implies absence of reflux for the flux chosen here. For $D a=10^{-5}$, when the wall is porous, there is a reflux layer adjacent to the wall, and the reflux zone widens with an increase in $D a$. Figure 9(ii) depicts the variation of $Q_{\psi}^{*}$ as a function of $\psi^{*}$ for different value of $\alpha$ fixing $\bar{Q}=0.9, \phi=0.6, n=1$, and $D a=10^{-4}$. It is seen here that there is no reflux for $\alpha=5$ where as for $\alpha=1,0.5$ reflux layer exists near the wall and it spreads towards the center line as $\alpha$ decreases. The effects of power-law index $n$ on reflux is shown in the Fig 9(iii) by fixing $\bar{Q}=0.9, \phi=0.6, D a=10^{-4}$ and $\alpha=0.5$. Reflux layer doesn't exist for small $n$ but for a value of $n$ close to unity reflux may exist and for large $n>1$ (shear thickening fluid) it spreads towards the center line. In the Fig. 9, the curves intersecting the axis of $Q_{\psi}^{*}$ corresponds to the flow giving trapping bolus which is discussed in Figs 5-7, and these are similar to qualitative curves given in [1]. The flux rate $\bar{Q}$ of Fig 9 is chosen to lie in the pumping range and the reflux layer for various parameters $D a, \alpha$ and $n$ widens as the corresponding pumping pressure is high. The limit on $\bar{Q}$ for reflux is obtained numerically when $\Delta p \geq 0$, and we find the reflux is absent in the entire copumping range irrespective of the presence of porous wall or non-Newtonian nature of the fluid.

\section{Conclusions}

The peristaltic transport of a power-law fluid in an axisymmetric tube having a poroflexible wall is studied under lubrication analysis. The effect of shear-thinning and shear-thickening properties of fluid are investigated on peristaltic pumping, trapping and reflux phenomena. Solutions are presented for two types namely Beavers-Joseph and Saffman slip boundary conditions. The peristalsis works as a pump against a greater pressure rise with the Saffman boundary conditions compared with Beavers-Joseph conditions for a shear thickening fluid than a shear thinning fluid or Newtonian fluid. Pumping characteristics are also discussed with a SSD wave form other than the usual sinusoidal one. It is observed that the time-mean flow becomes negative in free pumping for a shear-thickening fluid with a SSD expansion wave and the same is observed for a SSD contraction wave in the case of shear-thinning fluid. The trapped bolus for sinusoidal wave is observed to decrease as the fluid behavior index decreases, i.e. when it changes from shear-thickening to shear-thinning. 


\section{Acknowledgements}

The authors thank the referees for their useful suggestions. This work is partially funded from the grant DST/MS/110/99 by the Department of Science and Technology, Government of India.

\section{References}

[1] A.H. Shapiro, M.Y. Jaffrin, and S.L. Weinberg, Peristaltic pumping with long wavelengths at low Reynolds number, J. Fluid Mech., 37(1969), 799-825.

[2] M.Y. Jaffrin and A.H. Shapiro, Peristaltic pumping, Ann. Rev. Fluid Mech., 3(1971), 13-36.

[3] G. Böhme, and R. Friedrich, Peristaltic flow of viscoelastic liquids, J. Fluid Mech., 128(1983), 109-122.

[4] G. Radhakrishnamacharya, Long wavelength approximation to peristaltic motion of a power law fluid, Rheol. Acta, 21(1982), 30-35.

[5] L.P. Srivastava and V. P. Srivastava, Peristaltic transport of Blood: Casson model: II, J. Biomech., 17(1984), 821-830.

[6] D. Srinivasacharya, M. Mishra and A. R. Rao, Peristaltic pumping of a micropolar fluid in a tube, Acta Mechanica, 161(2003), 165-178.

[7] A. M. Siddiqui, A. Provost and W. H. Schwarz, Peristaltic pumping of a second-order fluid in a planar channel, Rheol. Acta, 30(1991), 249-262.

[8] A.M. Siddiqui and W.H. Schwarz, Peristaltic flow of a second-order fluid in tubes, J. NonNewtonian Fluid Mech., 53(1994), 257-284.

[9] L.M. Srivastava and V. P. Srivastava, V.P., Peristaltic transport of a power-law fluid: applications to the ductus efferentes of the reproductive tract, Rheol. Acta, 27(1988), 428-433.

[10] L.M. Srivastava and V. P, Srivastava, Peristaltic transport of a Non-Newtonian fluid: Applications to the vas deferens and small intestine, Annals Biomed. engng, 13(1985), 137-153.

[11] J.C. Misra and S.K. Pandey, A Mathematical model for Oesophegeal swallowing of a foodbolus, Math. Comput. model., 33(2001), 997-1009.

[12] S. Usha and A. Ramachandra Rao, Peristaltic transport of two-layered power-law fluids, Trans. ASME J. Biomech. engng., 119(1997), 483-488.

[13] J.B. Shukla and S.P. Gupta, Peristaltic transport of a power-law fluid with variable consistency, Trans. ASME J. Biomech.engng., 104(1982), 182-186. 
[14] S. Najaraian and H. Niroomand, Peristaltic transport of a power-law fluid with variable consistency, in Proc. 12th conference of the European Society of Biomechanics, Dublin, 2000, p. 308.

[15] J.R. Keener and J. Sneyd, Mathematical Physiology, Springer, New York, 1998, Chap. 21.

[16] G. W. Reese and H.J. Rath, A model of a combined porous peristaltic pumping system, in L. S. Srinath and M. Singh (Eds), Physiological Fluid Dynamics II, Proc. 2nd international conference on physiological fluid dynamics, IIT Madras, 1987, Tata McGraw-Hill, New Delhi, p. $190-195$.

[17] G.S. Beavers and D.D. Joseph, Boundary conditions at a naturally permeable wall, J. Fluid Mech. 30(1967), 197-207.

[18] P.G. Saffman, On the boundary conditions at the surface of a porous medium, Stud. Appl. Math. 1(1971), 93-101.

[19] R.B. Bird, W.E. Stewart and E.N. Lightfoot, Transport Phenomena, John Wiley \& Sons, Singapore, 1960.

[20] N.P. Cheremisinoff, Encyclopedia of Fluid Mechanics, vol-7: Rheology and Non-Newtonian flows, Gulf publishing Co., Houston, TX, 1988.

[21] A.M. Provost and W.H. Schwarz, A theoretical study of viscous effects in peristaltic pumping, J. Fluid. Mech. 279(1994), 177-195.

[22] A.B. Metzner and J.C. Reed, Flow of non-Newtonian fluids-correlation of the laminar, transition, and turbulent-flow regions, AIChE J., 1(4)(1955), 434-440.

[23] A.V. Shenoy, Non-Newtonian fluid heat transfer in porous media, Adv. Heat transfer, 24(1994), 101-190.

[24] A. Ramachandra Rao and S. Usha, Peristaltic transport of two immiscible viscous fluid in a circular tube, J. Fluid Mech., 298(1995), 271-285. 


\section{List of Figure Captions}

Figure 1. Schematic representation of peristaltic transport in an axisymmetric tube with a porous wall in a fixed frame.

Figure 2. The variation of $\bar{Q}$ with $\Delta p$ fixing $\phi=0.6, m=1$ and $n=1$, (i) for different $D a$ with fixed $\alpha=0.5$, (ii) for different $\alpha$ with fixed $D a=10^{-4}$. Solid lines and dashed lines correspond to Beavers-Joseph and Saffman models respectively.

Figure 3. The variation of $\Delta p_{0}$ with $n$ fixing $\phi=0.6, m=1$, (i) for different $D a$ with fixed $\alpha=0.5$, (ii) for different $\alpha$ with fixed $D a=10^{-4}$. Solid lines and dashed lines correspond to Beavers-Joseph and Saffman models respectively.

Figure 4. The variation of $\bar{Q}$ with $\Delta p$ fixing $\phi=0.4, \lambda_{c}=0.2, D a=0$ (no slip) for different $n$, (i) SSD contraction wave, (ii) SSD expansion wave, (iii) pumping characteristics for SSD expansion wave with $\phi=0.3, \lambda_{c}=0.2, m=1, D a=10^{-5}$, and $n=2$ for different $\alpha$.

Figure 5. The variation of $\Delta p_{0}$ with $\lambda_{c}$ fixing $D a=10^{-5}, \alpha=0.5, m=1, \phi=0.4$ for different $n$, (i) SSD contraction wave, (ii) SSD expansion wave.

Figure 6. Streamlines in the wave frame with $\bar{Q}=0.1, \alpha=0.5, \phi=0.4, n=1, m=1$, (i) $D a=0$, (ii) $D a=10^{-5}$, (iii) $D a=10^{-4}$.

Figure 7. Streamlines in the wave frame with $\bar{Q}=0.1, D a=10^{-4}, \phi=0.4, n=1, m=1,(\mathrm{i})$ $\alpha=0.5$, (ii) $\alpha=1$, (iii) $\alpha=2$.

Figure 8. Streamlines in the wave frame with $\bar{Q}=0.3, D a=10^{-4}, \phi=0.4, \alpha=0.5, m=1$, (i) $n=2$, (ii) $n=1$, (iii) $n=0.5$, (iv) $n=0.3$.

Figure 9. The variation of $Q_{\psi}^{*}$ versus $\psi^{*}$ with $\phi=0.6, m=1$, (i) for different $D a$ fixing $\bar{Q}=0.915, \alpha=0.5, n=1$, (ii) for different $\alpha$ fixing $\bar{Q}=0.9, D a=10^{-4}, n=1$, (iii) for different $n$ fixing $\bar{Q}=0.9, D a=10^{-4}, \alpha=0.5$. 


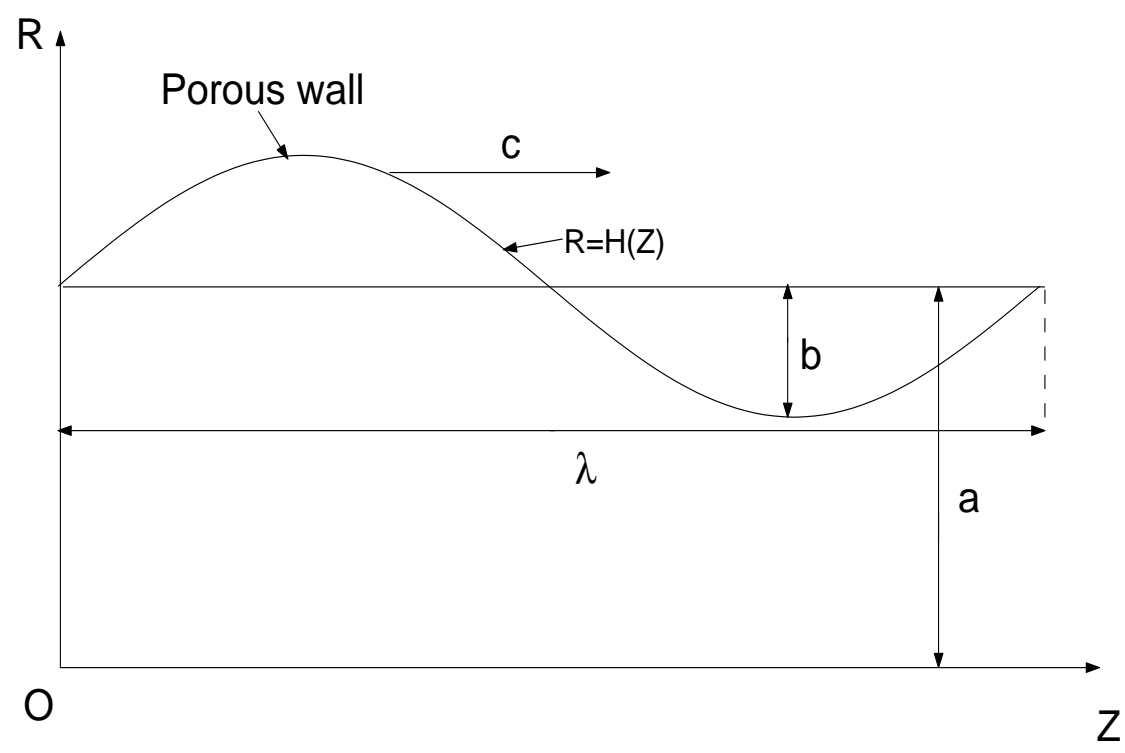

Figure 1: Schematic representation of peristaltic transport in an axisymmetric tube with a porous wall in a fixed frame.
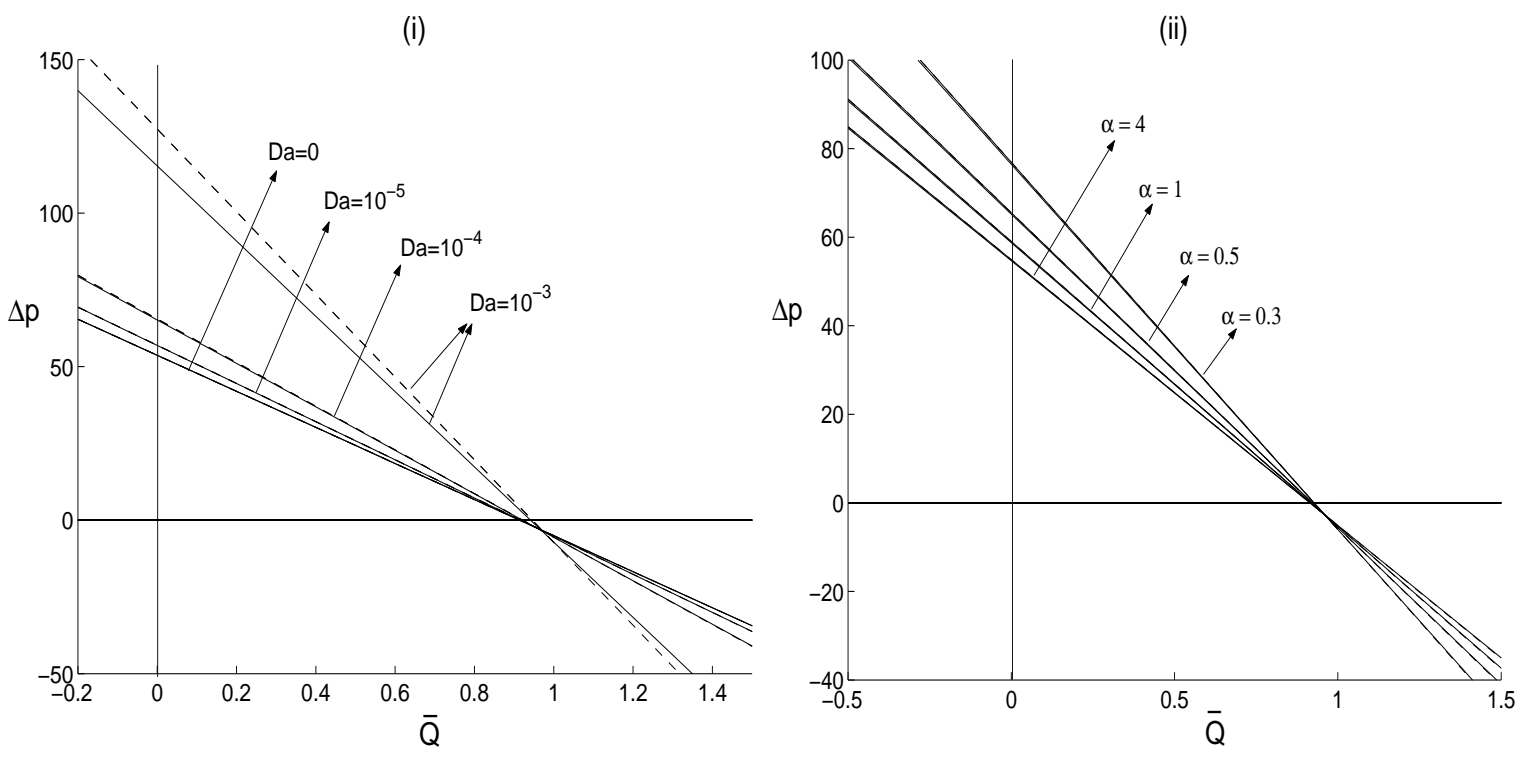

Figure 2: The variation of $\bar{Q}$ with $\Delta p$ fixing $\phi=0.6, m=1$ and $n=1$, (i) for different $D a$ with fixed $\alpha=0.5$, (ii) for different $\alpha$ with fixed $D a=10^{-4}$. Solid lines and dashed lines correspond to Beavers-Joseph and Saffman models respectively. 

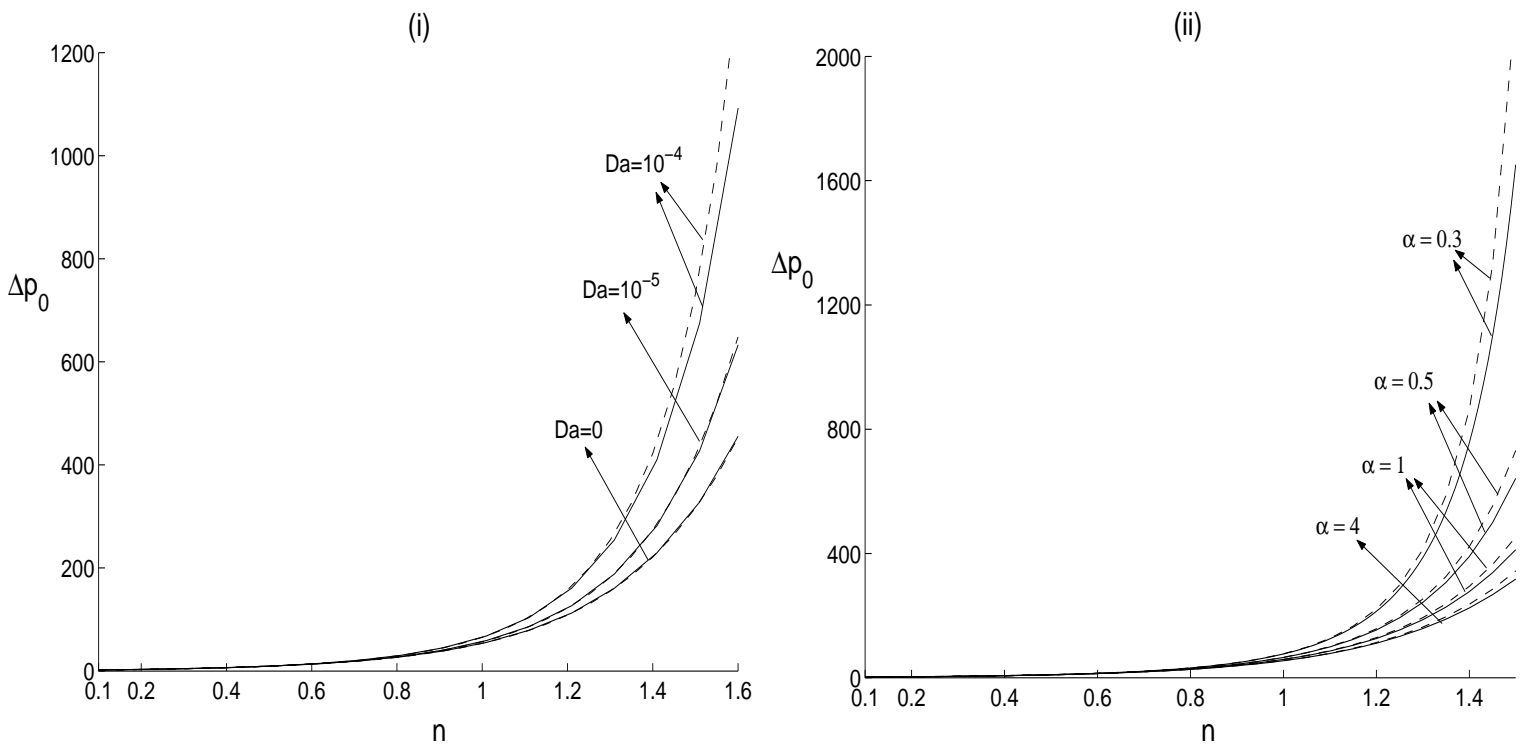

Figure 3: The variation of $\Delta p_{0}$ with $n$ fixing $\phi=0.6, m=1$, (i) for different $D a$ with fixed $\alpha=0.5$, (ii) for different $\alpha$ with fixed $D a=10^{-4}$. Solid lines and dashed lines correspond to Beavers-Joseph and Saffman models respectively. 
(i)
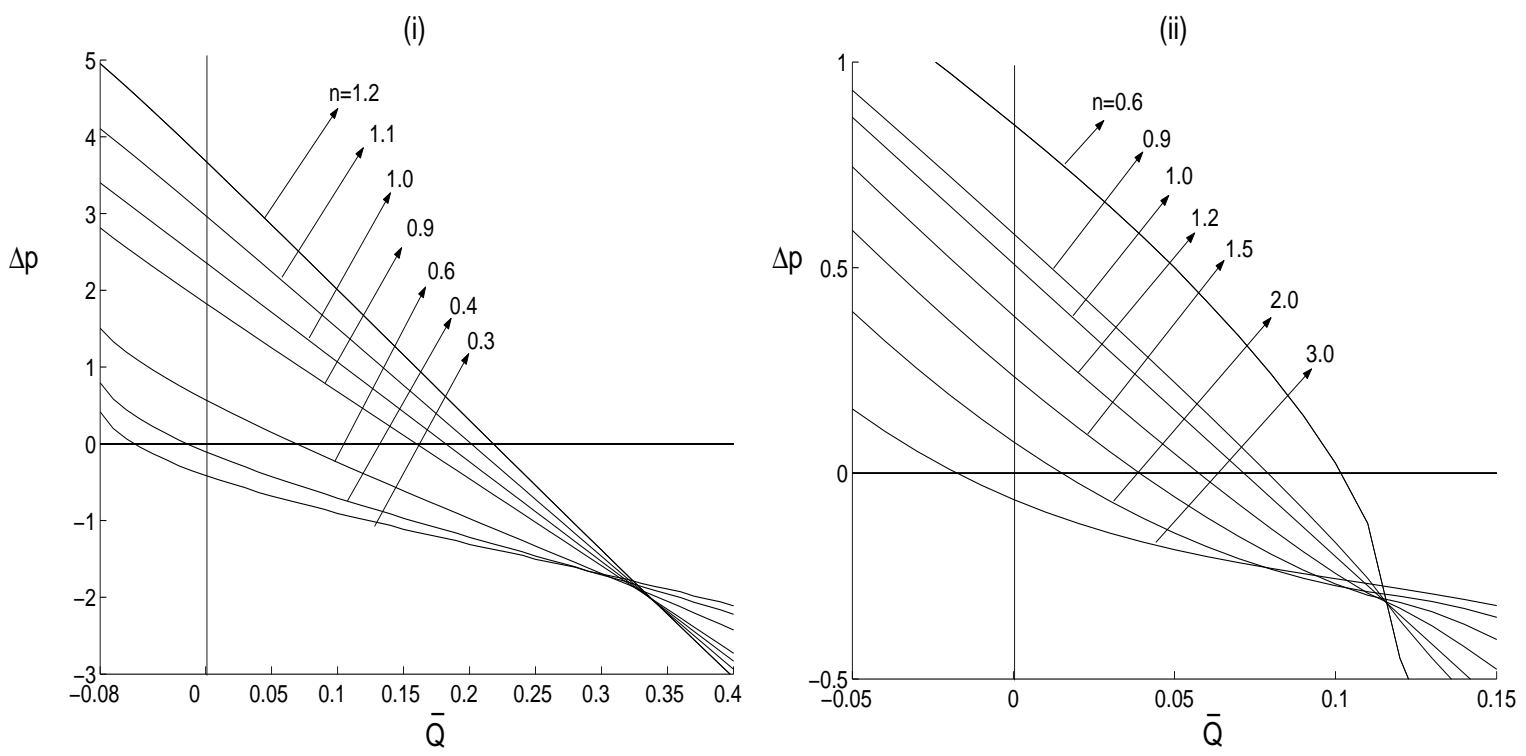

(iii)

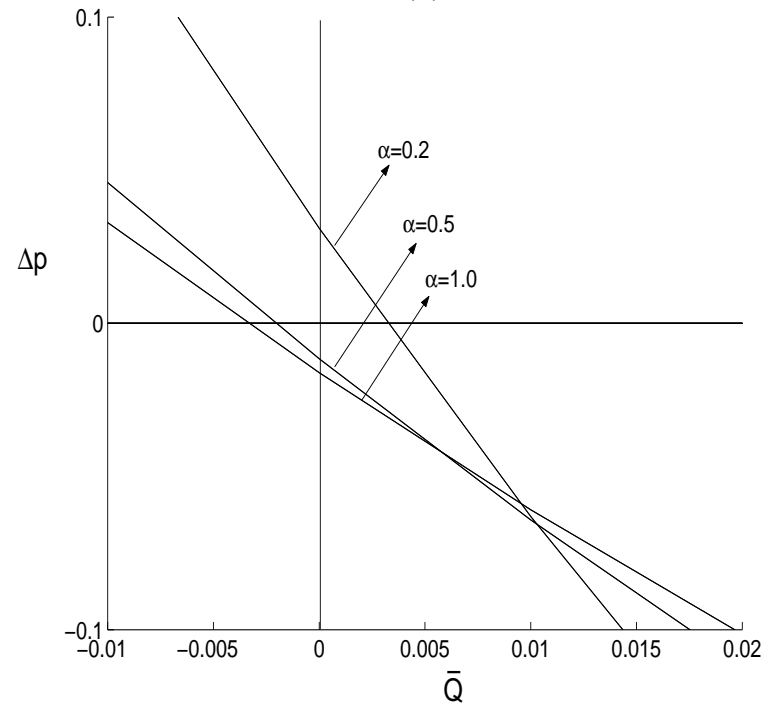

Figure 4: The variation of $\bar{Q}$ with $\Delta p$ fixing $\phi=0.4, \lambda_{c}=0.2, D a=0$ (no slip) for different $n$, (i) SSD contraction wave, (ii) SSD expansion wave, (iii) pumping characteristics for SSD expansion wave with $\phi=0.3, \lambda_{c}=0.2, m=1, D a=10^{-5}$ and $n=2$ for different $\alpha$. 
(i)

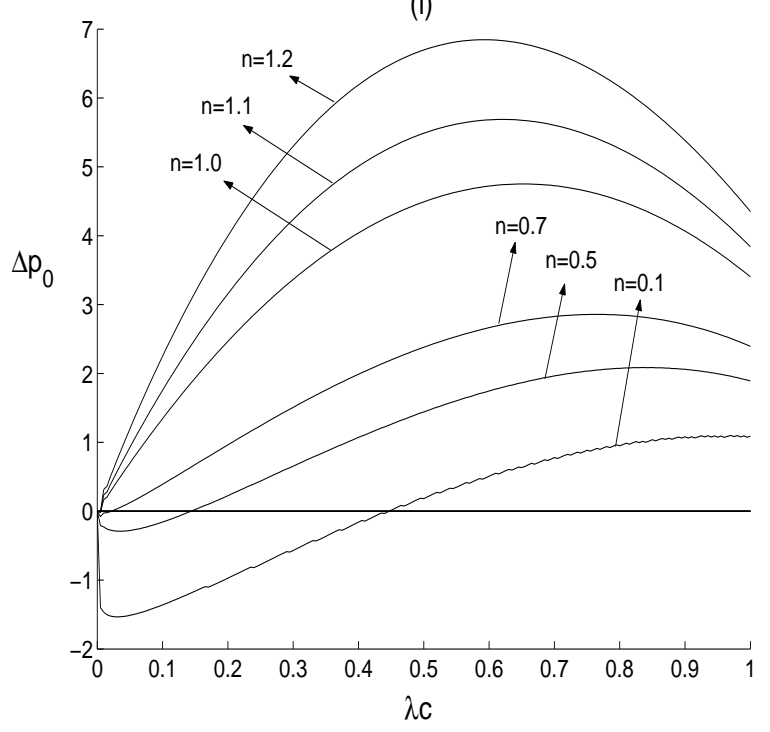

(ii)

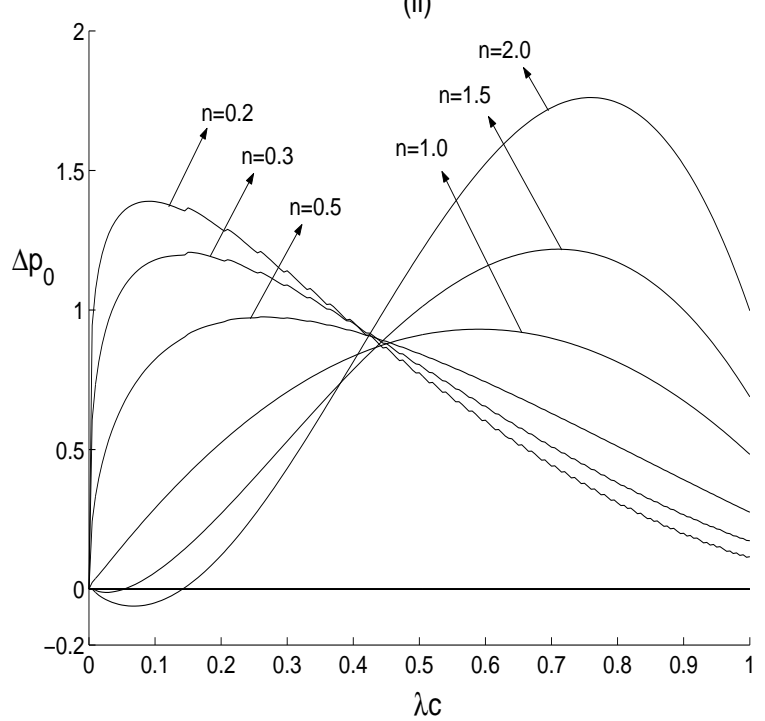

Figure 5: The variation of $\Delta p_{0}$ with $\lambda_{c}$ fixing $D a=10^{-5}, \alpha=0.5, m=1, \phi=0.4$ for different $n$, (i)SSD contraction wave, (ii) SSD expansion wave. 

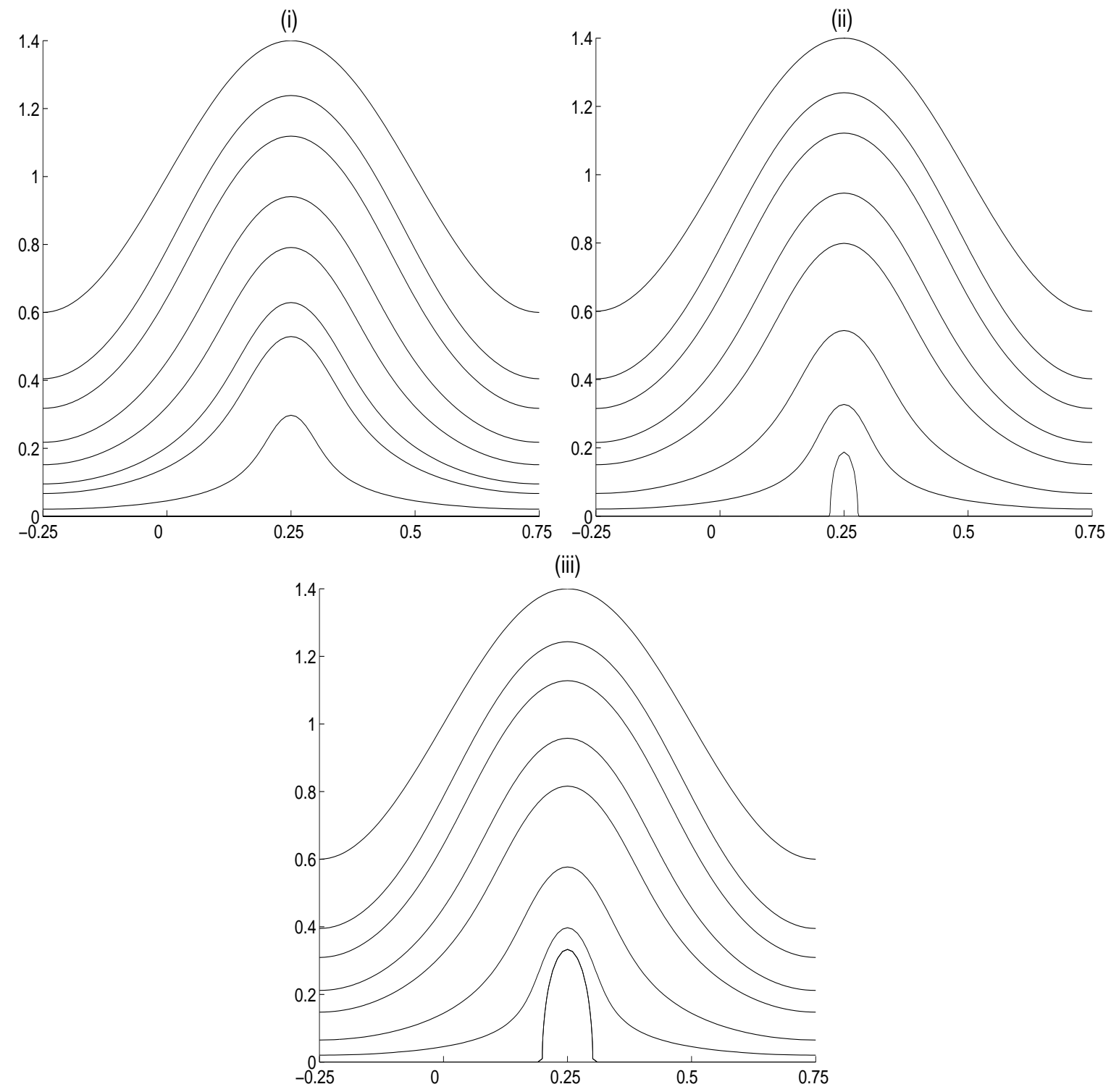

Figure 6: Streamlines in the wave frame with $\bar{Q}=0.1, \alpha=0.5, \phi=0.4, n=1, m=1$, (i) $D a=0$, (ii) $D a=10^{-5}$, (iii) $D a=10^{-4}$. 

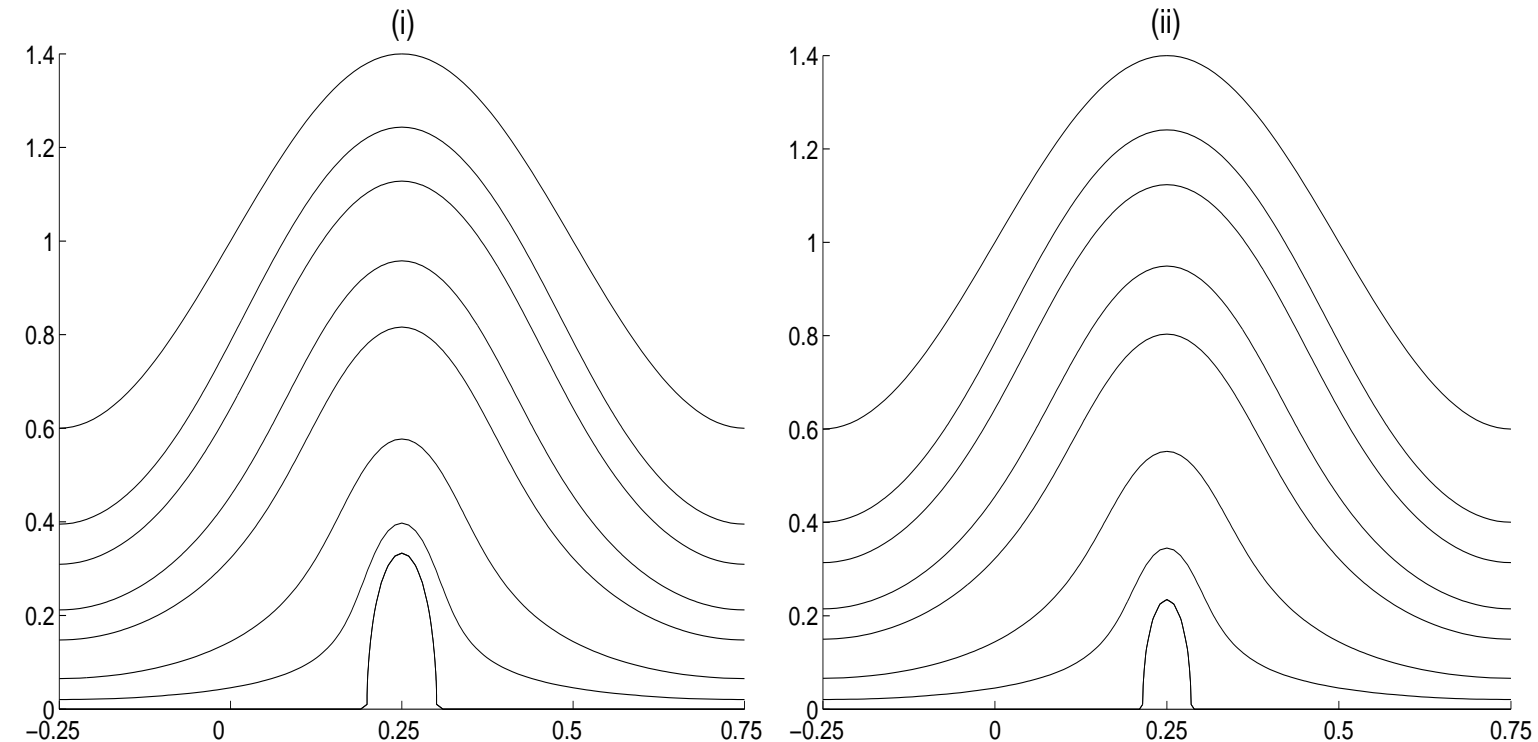

(iii)

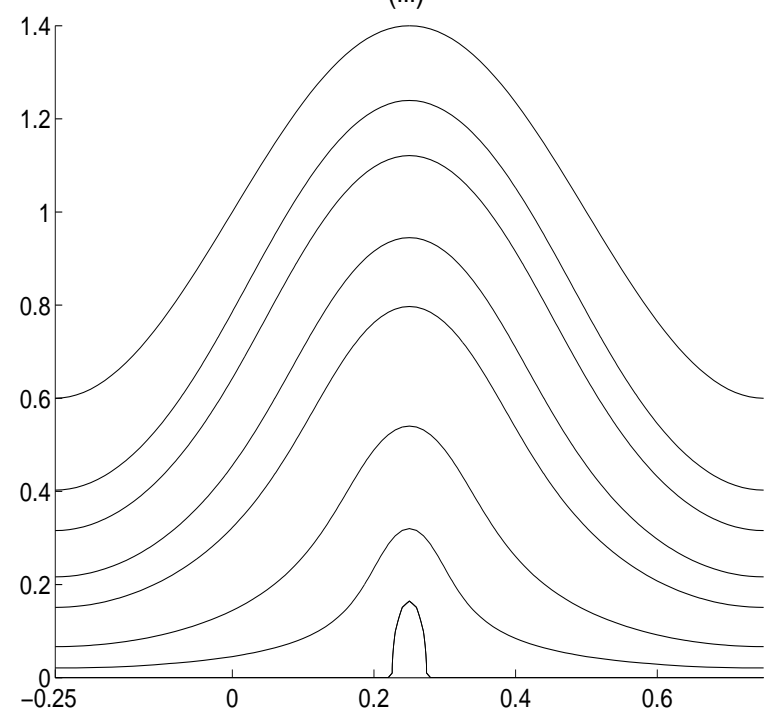

Figure 7: Streamlines in the wave frame with $\bar{Q}=0.1, D a=10^{-4}, \phi=0.4, n=1, m=1,(\mathrm{i})$ $\alpha=0.5$, (ii) $\alpha=1$, (iii) $\alpha=2$. 

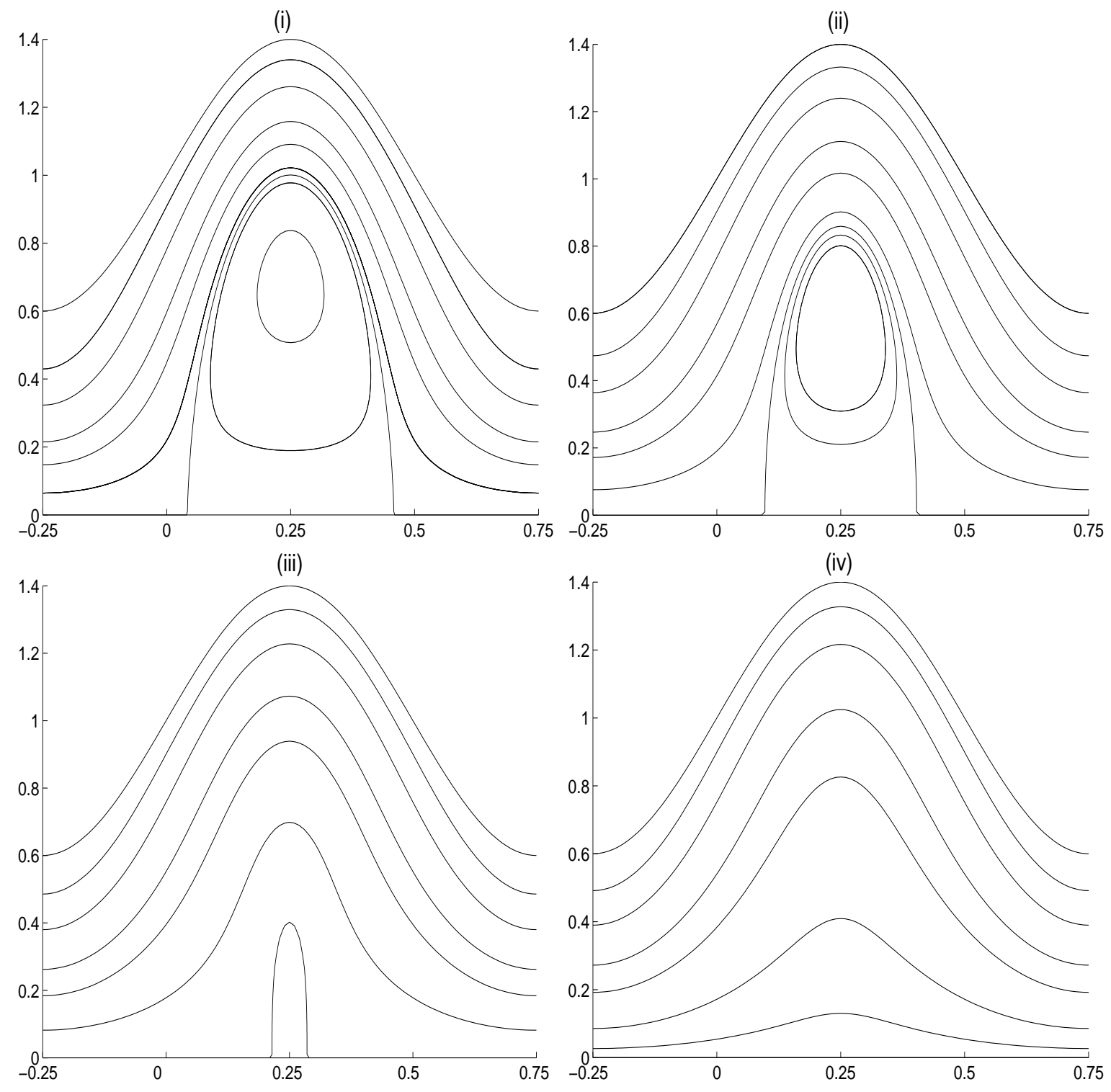

Figure 8: Streamlines in the wave frame with $\bar{Q}=0.3, D a=10^{-4}, \phi=0.4, \alpha=0.5, m=1$, (i) $n=2$, (ii) $n=1$, (iii) $n=0.5$, (iv) $n=0.3$. 

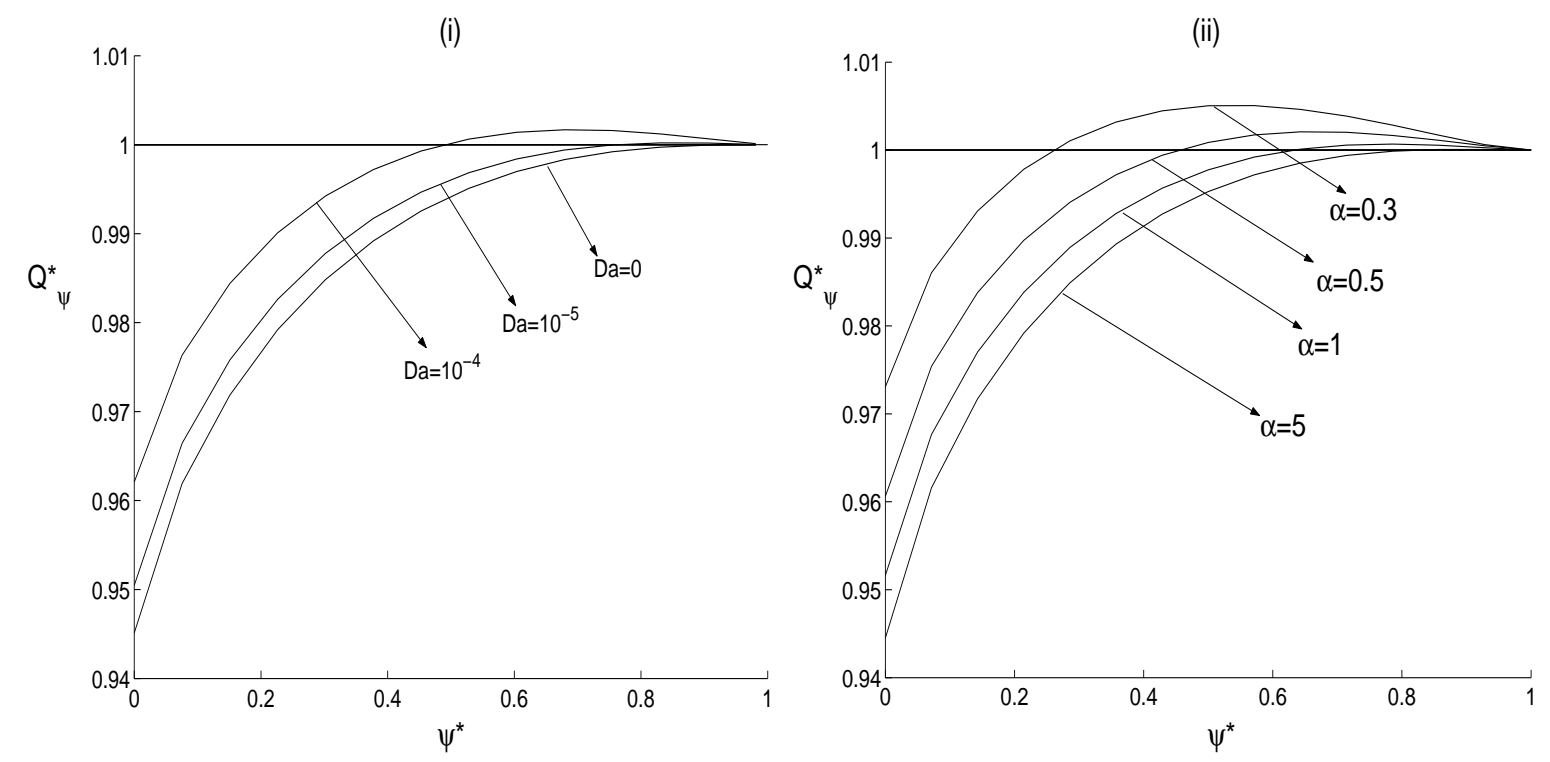

(iii)

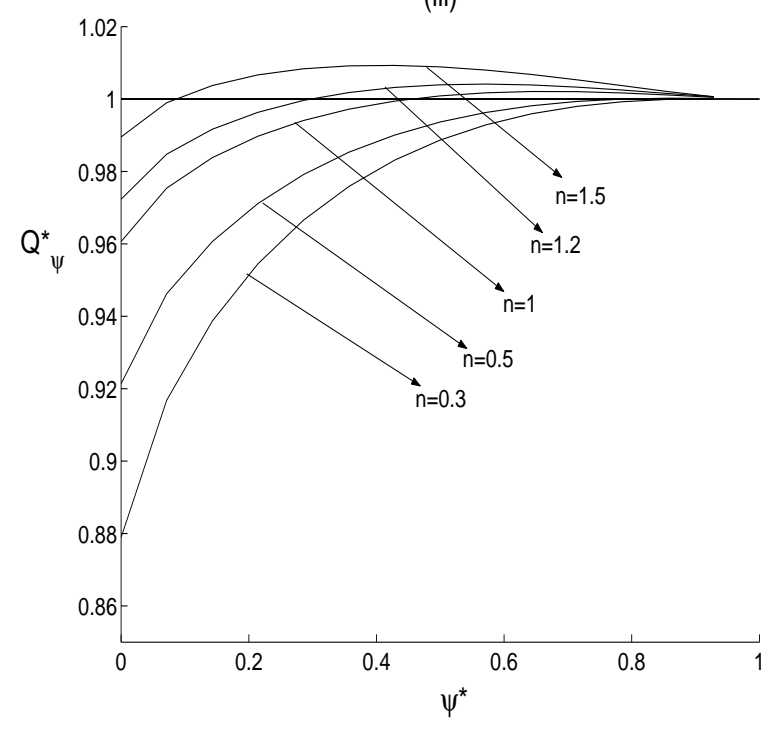

Figure 9: The variation of $Q_{\psi}^{*}$ versus $\psi^{*}$ with $\phi=0.6, m=1$, (i) for different $D a$ fixing $\bar{Q}=0.915, \alpha=0.5, n=1$, (ii) for different $\alpha$ fixing $\bar{Q}=0.9, D a=10^{-4}, n=1$, (iii) for different $n$ fixing $\bar{Q}=0.9, D a=10^{-4}, \alpha=0.5$. 